\title{
Blowout Bifurcation of Chaotic Saddles
}

\author{
TOMASZ KAPITANIAK ${ }^{\mathrm{a}, \mathrm{b}, *}$, YING-CHENG LAI $^{\mathrm{a}, \mathrm{c}}$ and CELSO GREBOGI ${ }^{\mathrm{a}, \mathrm{d}}$ \\ ${ }^{a}$ Institute for Plasma Research, University of Maryland, College Park, MD 20742, USA; ${ }^{\mathrm{b}}$ Division of Dynamics, \\ Technical University of Lodz, Stefanowskiego 1/15, 90-924 Lodz, Poland; ${ }^{\mathrm{c}}$ Departments of Physics and Astronomy \\ and of Mathematics, University of Kansas, Lawrence, KS 66045, USA; ${ }^{\mathrm{d}}$ Department of Mathematics, Institute for \\ Physical Science and Technology, University of Maryland, College Park, MD 20742, USA
}

(Received 4 February 1999)

\begin{abstract}
Chaotic saddles are nonattracting dynamical invariant sets that can lead to a variety of physical phenomena. We describe the blowout bifurcation of chaotic saddles located in the symmetric invariant manifold of coupled systems and discuss dynamical phenomena associated with this bifurcation.
\end{abstract}

Keywords: Nonattracting sets, Riddled basins, Blowout bifurcation, Chaos synchronization

Blowout bifurcation $[1,2]$ in chaotic dynamical systems occurs when the chaotic attractor, located in some invariant subspace, becomes transversally unstable. Let $\mathbf{S}$ be the invariant subspace in which there is a chaotic attractor $\mathcal{A}$ and $\mathbf{T}$ the subspace transverse to it. Since $\mathbf{S}$ is invariant, initial conditions in $\mathbf{S}$ result in trajectories that are attracted to $\mathcal{A}$ and remain in $\mathbf{S}$ forever. Whether the chaotic attractor $\mathcal{A}$ is also an attractor in full phase space depends on the sign of the largest Lyapunov exponent $\Lambda_{T}$ computed for the trajectories on $\mathcal{A}$ with respect to perturbations in the subspace $\mathbf{T}$. If $\Lambda_{T}$ is negative, $\mathcal{A}$ attracts trajectories transversally in the vicinity of $\mathbf{S}$ and $\mathcal{A}$ is an attractor in the full phase space. When $\Lambda_{T}$ is positive, trajectories in the neighbourhood of $\mathcal{A}$ are repelled away from $\mathbf{S}$, and chaotic attractor $\mathcal{A}$ is not an attractor in the full phase space. Blowout bifurcation occurs when $\Lambda_{T}$ changes sign from negative to positive.

Some distinct physical phenomena are associated with the blowout bifurcation. Near the bifurcation point when $\Lambda_{T}$ is still negative, the basin of chaotic attractor $\mathcal{A}$ is typically riddled [3-5]. When there are (are not) other attractors in the neighbourhood of $\mathbf{S}$ riddling is global (local) [4]. Global riddling means that for every point in the neighbourhood of $\mathcal{A}$ there are points arbitrarily nearby that belong to the basins of other attractor. When riddling is local for every point in the neighbourhood of $\mathcal{A}$ there are points arbitrarily nearby which leave this neighbourhood temporarily but finally are attracted to $\mathcal{A}$. Above the blowout bifurcation when $\Lambda_{T}$ is positive but small one can observe on-off intermittency.

\footnotetext{
* Corresponding author. Division of Dynamics, Technical University of Lodz, Stefanowskiego 1/15, 90-924 Lodz, Poland.
} 
Suppose that the dynamical system $X_{n+1}=$ $F\left(X_{n}, \rho, d\right)$, where $X \in \mathcal{R}^{n}, \rho$ and $d$ are constants, has a chaotic attractor $\mathcal{A}$ for fixed $\rho$ and $d \in D=\left[d_{1}, d_{2}\right]$ and undergoes a blowout bifurcation at $d=d_{c} \in D$. In this paper we address the question on what kind of dynamics is exhibited by this system when small changes in the parameter $\rho$ transform the chaotic attractor to a chaotic saddle $\mathcal{S}$ and a periodic attractor $\mathcal{P}$ with both $\mathcal{S}$ and $\mathcal{P}$ located on the invariant manifold $\mathbf{S}$. We describe the blowout bifurcation of the chaotic saddle located in the invariant subspace of two coupled one-dimensional maps and show that, shortly before the blowout bifurcation in the neighbourhood of the chaotic saddle, one can observe phenomena equivalent to that observed for the case when there exists a chaotic attractor in the invariant subspace [8].

As an example of a system that exhibits a blowout bifurcation of a chaotic saddle we consider the system consisting of two coupled logistic maps

$$
\begin{aligned}
x_{n+1} & =f\left(x_{n}\right)+d\left(y_{n}-x_{n}\right) \\
& =\rho x_{n}\left(1-x_{n}\right)+d\left(y_{n}-x_{n}\right), \\
y_{n+1} & =f\left(y_{n}\right)+d\left(x_{n}-y_{n}\right) \\
& =\rho y_{n}\left(1-y_{n}\right)+d\left(x_{n}-y_{n}\right),
\end{aligned}
$$

where $\rho, d \in \mathcal{R}$ are constants. The primary reason for choosing two-dimensional maps to illustrate our results is that for such systems, there exists a procedure, the Proper-Interior-Maximum triple (PIM-triple) procedure [7], for computing an arbitrarily long trajectory on a chaotic saddle with high precision. We are not aware of any procedure that can be utilized to compute trajectories on chaotic saddles in higher dimensions.

Dynamics of the system (1), when $\rho$ is fixed at the values for which the one-dimensional logistic map exhibits chaotic behaviour, has recently been studied by Maistrenko et al. [5]. In those studies, the regions of different types of stability of chaotic attractors located at invariant manifold, $x=y$, are described. Particularly, for $\rho=3.64$ and $d=-1.1$, system (1) has a chaotic attractor on the invariant manifold and when $d$ is decreased this attractor undergoes blowout bifurcation for $d_{\mathrm{c}}=-0.92$. In our numerical studies, we assume $\rho$ to be equal 3.63 , i.e., the system (1) has a chaotic saddle and a period-6 attractor at the invariant manifold. We study what kind of dynamical phenomena can be observed when $d$ is slowly varied through the blowout bifurcation point $d_{\mathrm{c}}$.

We now argue that the blowout bifurcation of the chaotic saddle can occur when the transverse Lyapunov exponent of the typical trajectory on chaotic saddle $\Lambda_{T}^{\mathrm{s}}$ passes through zero from the negative side as the coupling $\epsilon$ changes. We recall that a chaotic saddle is globally nonattracting and it has a basin of attraction of vanishing volume in the phase space. Nonetheless, a conditionally invariant measure can still be defined on the saddle. A conditionally invariant measure on a chaotic saddle can be defined as follows [9]. Imagine that we enclose the saddle by a cube $C$ in the phase space and we sprinkle a very large number $N(0)$ of initial conditions uniformly in the cube. The number of trajectories that still remain in $C$ is: $N(t) \approx N(0) \mathrm{e}^{-t / \tau}$, where $\tau$ is the average lifetime of the chaotic saddle. Let $N_{0}(\eta, t, C)$ be the number of trajectories that are in $C$ at time $\eta t$, where $0<\eta<1$. The conditionally invariant measure is defined to be:

$$
\mu_{0}(C)=\lim _{t \rightarrow+\infty} \lim _{n(0) \rightarrow \infty} \frac{N_{0}(\eta, t, C)}{N(t)} .
$$

Consider a trajectory on the chaotic saddle in $\mathbf{S}$ with respect to the conditionally invariant measure: call it a conditional trajectory. By continuity, a trajectory in the vicinity of $\mathbf{S}$ in the transverse subspaces is also a conditional trajectory. For $\Lambda_{T}^{\mathrm{s}}<0$, the conditionally invariant measure on the chaotic saddle is transverse stable. That is, the chaotic saddle tends to attract nearby conditional trajectories in the transverse directions. In this case, the chaotic saddle is confined within the invariant manifold and it is isolated from the remaining of the phase space. For $\Lambda_{T}^{\mathrm{s}}>0$, the conditionally invariant measure on the chaotic saddle becomes unstable. In this case, a conditional trajectory in the neighbourhood of the chaotic saddle is typically repelled away from it asymptotically. Since the 
trajectory is conditional, it remains conditional under the dynamics even though it is no longer confined within $\mathbf{S}$. The corresponding measure that supports the trajectory must be conditional and, hence, it defines a chaotic saddle that extends beyond the invariant manifold $\mathbf{S}$ in the transverse subspaces. As a consequence, a sudden enlargement, or a metamorphosis, of the chaotic saddle occurs as $\Lambda_{T}^{\mathrm{s}}$ becomes positive. The newly acquired infinite pieces of the chaotic saddle do not exist before the bifurcation: they are created at the bifurcation when the chaotic saddle in $\mathbf{S}$ becomes transversely unstable.

In Fig. 1 we show the plot of transverse Lyapunow exponents

$$
\Lambda_{T}=\lim _{N \rightarrow \infty} \frac{1}{N} \sum_{n=1}^{N} \ln \left|f^{\prime}\left(x_{n}\right)-2 d\right|
$$

for a period-6 trajectory $\Lambda_{T}^{\mathrm{p}}$ and typical trajectory on chaotic saddle $\Lambda_{T}^{\mathrm{s}}$.

With decreasing $d$, one observes that the period-6 attractor becomes transversally unstable (at $d_{2}=$
-1.61 ) before the chaotic saddle (at $d_{1}=-1.65$ ). In the region $\mathrm{I}$, the chaotic saddle is located in the invariant subspace $x=y$ and a typical trajectory spends some time in the neighbourhood of the invariant subspace before finally escaping to infinity. In this case, the invariant manifold $\mathbf{S}$ is weakly transversally unstable as in the case of a chaotic attractor in $\mathbf{S}$ [8]. At $d_{1}$, the chaotic saddle undergoes a blowout bifurcation in which it is destroyed and a typical trajectory quickly escapes to infinity.

In the region II, the chaotic saddle in the invariant manifold is transversally weakly stable, but there is a finite measure set of trajectories originated in the vicinity of the chaotic saddle $\mathcal{S}$ which leaves the neighbourhood of $\mathbf{S}$ in the transverse direction but, after some time, this set returns to the invariant subspace and tends to the period-6 attractor $\mathcal{P}$. This leads to the phenomenon of local riddling. In the case of a chaotic saddle, the mechanism for local riddling is similar to the one known for the case of chaotic attractor. The only difference is that trajectories, after leaving the

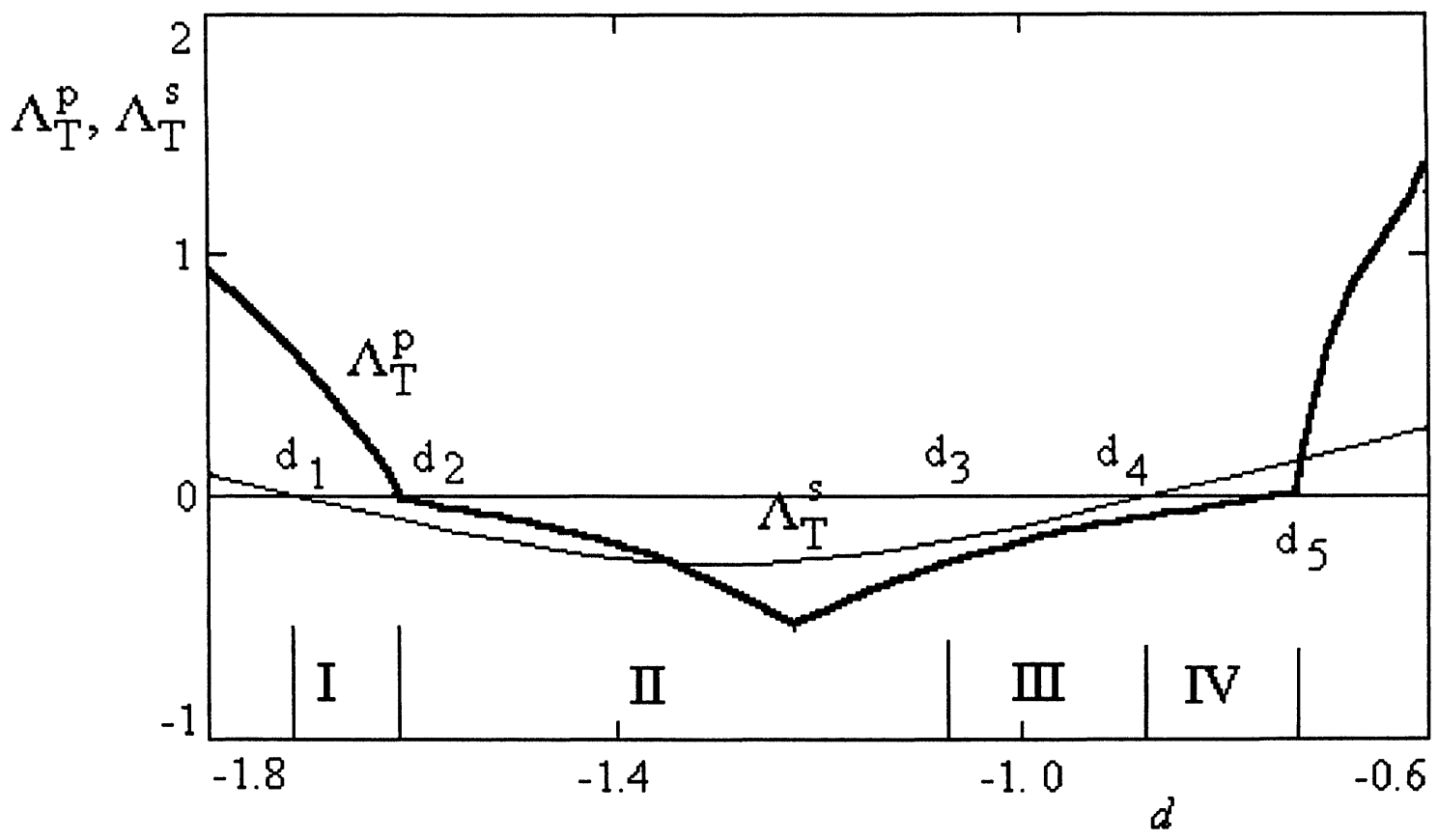

FIGURE 1 Transverse Lyapunov exponents $\Lambda_{T}^{\mathrm{p}}$ for the period-6 attractor and $\Lambda_{T}^{\mathrm{s}}$ for the chaotic saddle versus $d ; \rho=3.63$. 
neighbourhood of the chaotic saddle, return to the invariant subspace not to the chaotic saddle but to the basin of attraction of the period- 6 attractor. In Fig. 2(a), we show the period-6 attractor (indicated by crosses), its basin of attraction (shown in red) and the chaotic saddle (yellow).

At $d_{3}=-1.06$, system (1) undergoes a transition from local to global riddling. After this transition, some of the system trajectories which leaving the neighbourhood of the chaotic saddle never return to the invariant subspace but escape to infinity. Structure of the basin of attraction of the period- 6 attractor, shown in Fig. 2(b) $(d=-1.05)$, suggests that in the neighbourhood of the chaotic saddle (indicated in yellow) there is a positive Lebesgue measure set of points which asymptotes to infinity.

Finally, at $d_{4}=-0.92$, the chaotic saddle in the invariant manifold undergoes a blowout bifurcation in which it is transformed to a two-dimensional chaotic saddle as shown in Fig. 2(c). As a result of this bifurcation, most of the trajectories, after some evolution on the chaotic saddle, escape to infinity and now the basin of period- 6 attractor is much smaller. After the blowout bifurcation (Fig. 2(c)), the chaotic saddle appears to spread out in the phase space of system (1) with infinitely many new pieces. A detailed structure of the chaotic saddle, after this bifurcation, can be seen in Fig. 3(c) and (d), where enlargements of the chaotic saddle of Fig. 2(c) are shown. At the blowout bifurcation, there is a sudden enlargement of the chaotic saddle (its fractal dimension is doubled). We discuss this phenomenon in details in [6].

The period-6 attractor becomes transversally unstable at $d_{5}=-0.76$ and for larger values of $d$, all system trajectories asymptote to infinity.

Our example shows that the chaotic saddle located in the invariant subspace of the coupled systems can be characterized by four types of transverse stability (instability):

(i) When the transverse Lyapunov exponent of the chaotic saddle $\Lambda_{T}^{\mathrm{s}}$ is negative for all trajectories on $\mathcal{S}$, the chaotic saddle is strongly transversally stable.
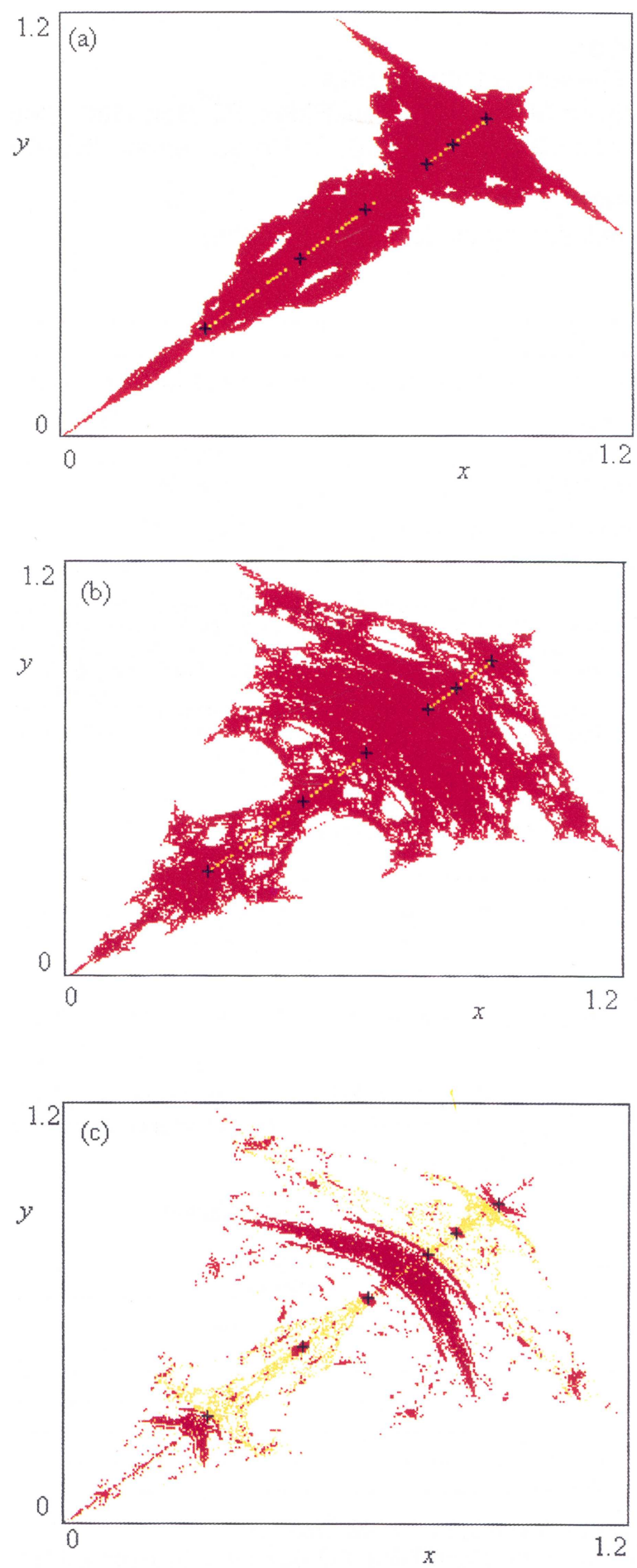

FIGURE 2 Period-6 attractor (crosses), its basin of attraction (red) and chaotic saddle (yellow) of system (1), $\rho=3.63$ : (a) $d=-1.4$, (b) $d=-1.05$, (c) $d=-0.9$. (See Color Plate I.) 

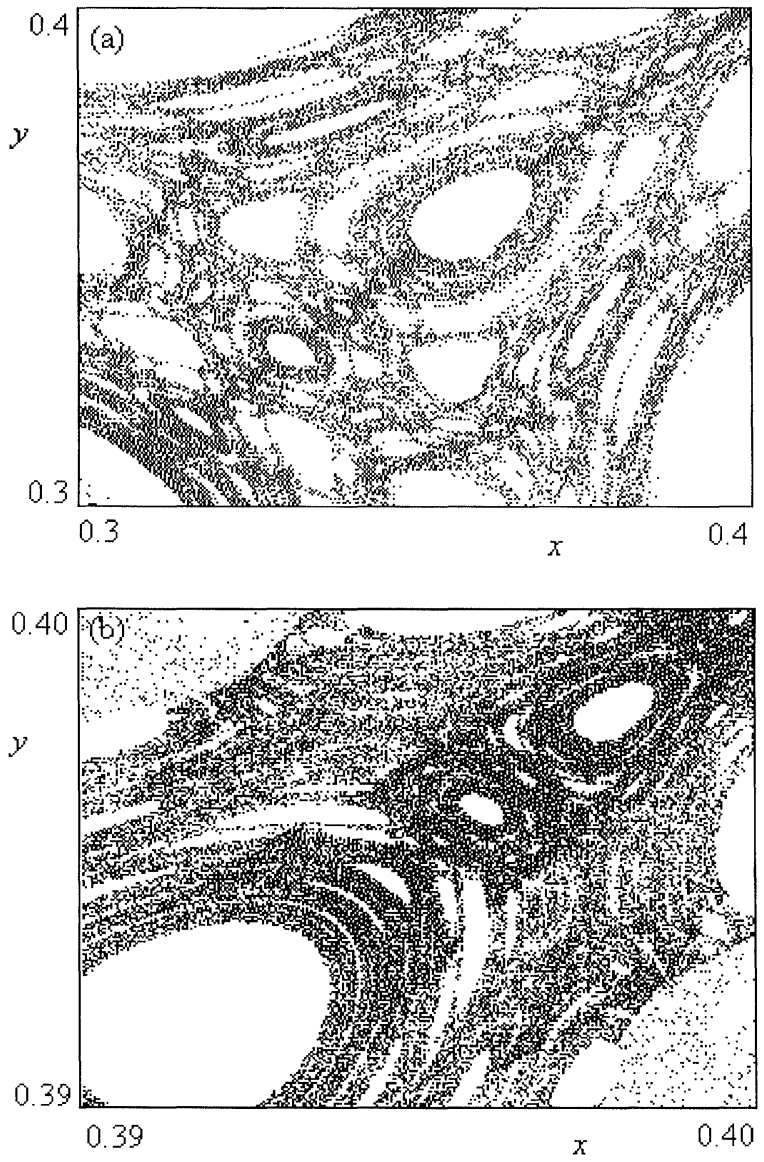

FIGURE 3 Detailed structure of the chaotic saddle after the blowout bifurcation (enlargements of Fig. 2(c)).

(ii) When $\Lambda_{T}^{\mathrm{s}}$ is negative for most of the trajectories but there exist trajectories for which transverse Lyapunov exponent is positive, the chaotic saddle is transversally weakly stable.

(iii) When $\Lambda_{T}^{\mathrm{s}}$ is positive for most of the trajectories on $\mathcal{S}$ but there are trajectories for which it is negative, the chaotic saddle is transversally weakly unstable.

(iv) When $\Lambda_{T}^{\mathrm{s}}$ is positive for all trajectories on $\mathcal{S}$, the chaotic saddle is transversally strongly unstable.

In the blowout bifurcation of the chaotic saddle, infinitely many orbits are involved, i.e., it occurs when the number of trajectories on the chaotic saddle, which is transversally unstable, is larger than the number of those which are transversally stable. The analogy with the mechanism of the blowout bifurcation of chaotic attractors [2] is straightforward.

In summary, we have studied the blowout bifurcation of a chaotic saddle. We stress that the model system Eq. (1) was used only for the purpose of illustrating the blowout bifurcation of the chaotic saddle. The logistic map used to describe the dynamics in the invariant space is considered as a paradigm in the study of nonlinear systems. We believe that our results are typical for a class of two coupled chaotic systems.

T.K. acknowledges the Fulbright Fellowship. Y.C.L. was supported by NSF under Grant No. PHY-9722156 and by AFOSR under Grant No. F49620-98-1-0400. This work was also supported by DOE (Mathematical, Information, and Computation Sciences Division, High Performance Computing and Communication Program) and by a joint NSF/CNPg grant.

\section{References}

[1] P. Ashwin, J. Buescu and I.N. Stewart, Phys. Lett. A 193, 126 (1994); ibid, Nonlinearity 9, 703 (1996); P. Ashwin, P.J. Aston and M. Nicol, Physica D, 111, 81 (1998).

[2] Y. Nagai and Y.-Ch. Lai, Phys. Rev. E 55, R1251 (1997); Y.-Ch. Lai, Phys. Rev. E 56, 1407 (1997); Y. Nagai and Y.-Ch. Lai, Phys. Rev. E 56, 4031 (1997).

[3] J.C. Alexander, I. Kan, J.A. Yorke and Z. You, Int. J. Bif. Chaos, 2, 795 (1992); J.F. Heagy, T. Carroll and L. Pecora, Phys. Rev. Lett. 73, 3528 (1994); Y.-C. Lai and C. Grebogi, Phys. Rev. E 52, R3313 (1995).

[4] Y. Maistrenko, T. Kapitaniak and P. Szuminski, Phys. Rev. E 56, 6393 (1997); T. Kapitaniak, Y. Maistrenko, A. Stefanski and J. Brindley, Phys. Rev. E 57, R6253 (1998)

[5] Y. Maistrenko, V. Maistrenko, A. Popovich and E. Mosekilde, Phys. Rev. Lett. 80, 1638 (1998); ibid Phys. Rev. E 57, 2713 (1998).

[6] T. Kapitaniak, Y.-C. Lai and C. Grebogi, preprint (1998).

[7] H.E. Nusse and J.A. Yorke, Physica D 36, 137 (1989).

[8] Y. Maistrenko and T. Kapitaniak, Phys. Rev. E 54, 3285 (1996); T. Kapitaniak and Y. Maistrenko, Chaos, Solitons and Fractals 9, 271 (1998).

[9] G.H. Hsu, E. Ott and C. Grebogi, Phys. Lett. A 127, 199 (1988). 


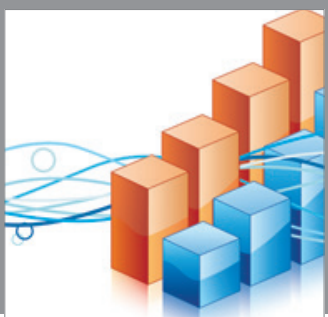

Advances in

Operations Research

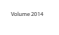

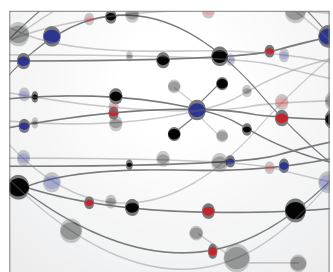

\section{The Scientific} World Journal
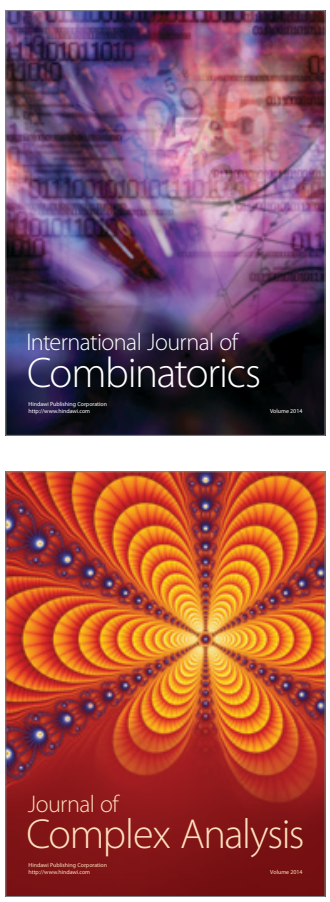

International Journal of

Mathematics and

Mathematical

Sciences
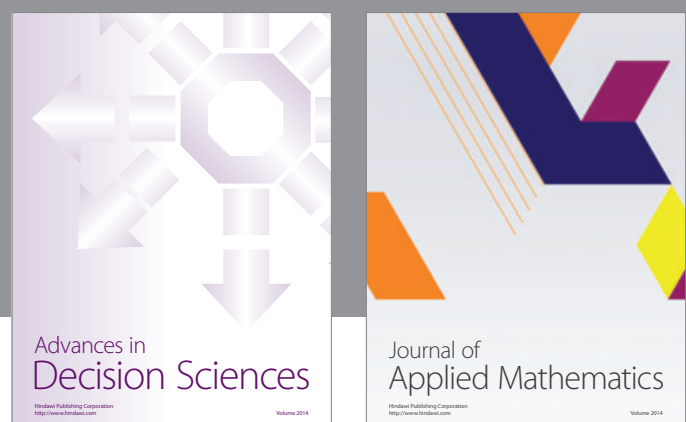

Journal of

Applied Mathematics
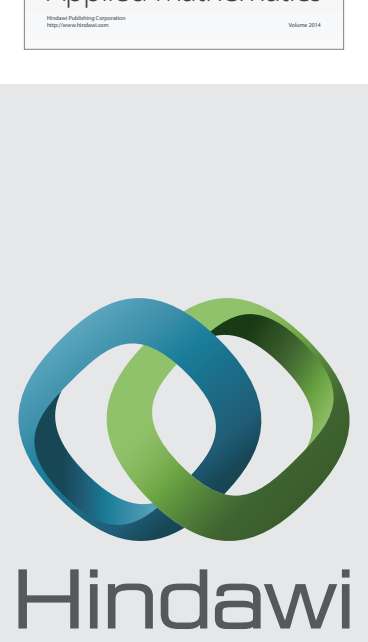

Submit your manuscripts at http://www.hindawi.com
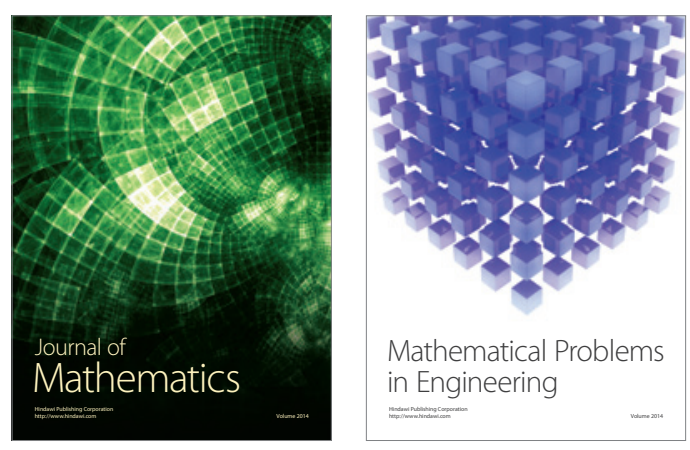

Mathematical Problems in Engineering
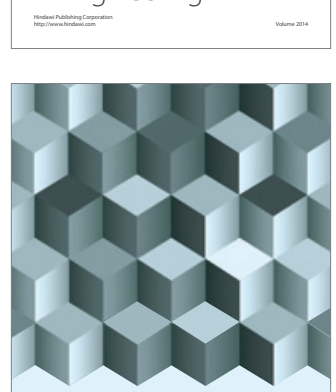

Journal of

Function Spaces
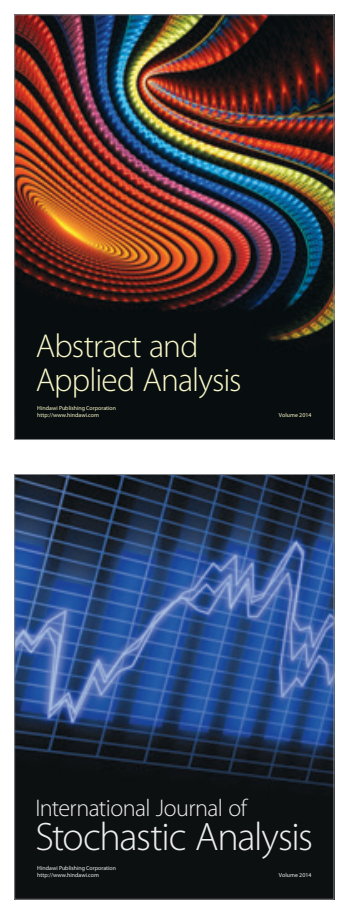

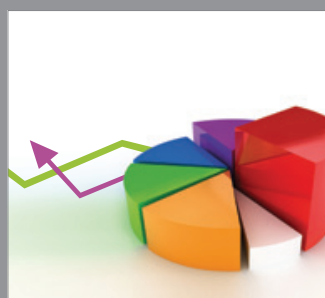

ournal of

Probability and Statistics

Promensencen
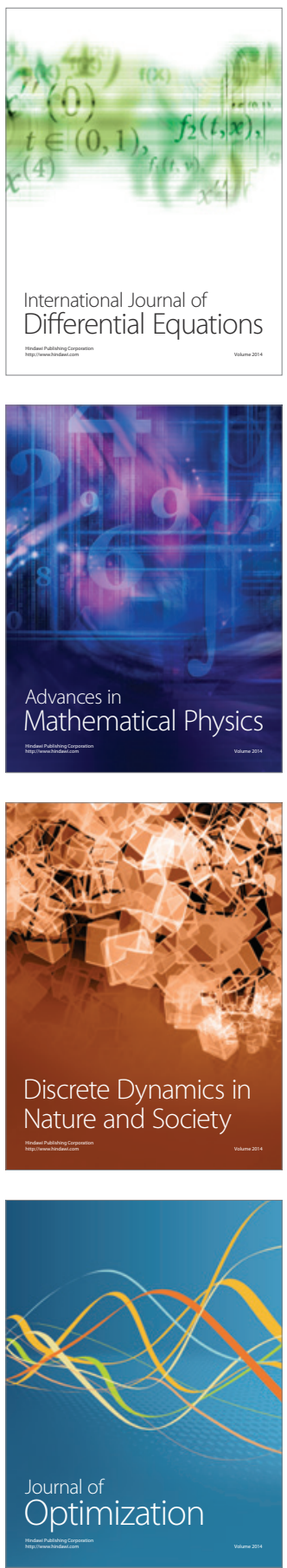